\title{
Laws and Models in a Theory of Idealization $\dagger$
}

\author{
Chuang Liu
}

We seem to trace [in King Lear] ... the tendency of imagination to analyse and abstract, to decompose human nature into its constituent factors, and then to construct beings in whom one or more of these factors is absent or atrophied or only incipient.

A.C. Bradley, Shakespearean Tragedy, p.264.

1. Introduction. The central challenge of modern science is to discover invariant laws of nature in a sea of ephemeral phenomena. I say 'modern science' because ancient astronomers were not dealing with the ephemera among the starry spheres, and by 'invariant', instead of 'universal', I mean laws that apply in diverse circumstances. This is especially true ever since the kinetic theory of gas dawned the search for invariants in the murky realm of thermo-statistical phenomena. Just as experimental design or control is essential to experimental sciences, idealization -- the counterpart of that -- is indispensable for theory construction.

This paper may be seen as a continuation of the work done in (Liu 1999), where a critique of the traditional theories of approximation and idealization is given and a possible alternative suggested. But to make it self-contained, a brief summary of that critique is given here first; and after identifying one of the major roles of idealization as detaching component processes or systems from joints of nature, a detailed analysis is given of idealized laws -- which are discoverable and/or applicable -- in such processes and systems (i.e. idealized model systems). Then, arguments are given for the inclusion of dispositional properties in the class of admissible properties for laws; and such an inclusion turns out to be crucial to our understanding of the relation between idealized models and the laws they 'produce' or 'accommodate'. And then I argue that idealized laws so produced or accommodated in the models are either true simpliciter or 
only approximately true but not so because of the idealizations in question. Finally I compare my theory with some existing theories of laws of nature.

2. Idealization: a brief summary (cf. Liu 1999). Idealization usually refers to acts of theorizing by which ideal-condition claims are stipulated and idealized claims obtained as a result, where ideal-condition claims are of physically possible but non-actual conditions and idealized claims those that are true under the conditions. The existing theories (or conceptions) of idealization further take such acts to be ones which either neglect the negligibles -- the idealcondition claims are approximately true -- or approbate approximation -- when the ideal-condition claims are relaxed towards the truth, the lawlike statements become more and more approximately true. Here, $\mathrm{p}$ is approximately true if the possible world in which it is true is factually close to our world. The factual distance may be most simply defined as a metric in a predicate space that gives exhaustive description of all the possible relevant states of affairs (cf. Hilpinen 1976; Niiniluoto 1984, 1998).

To make the above idea a bit more precise (cf. Laymon 1980, 1985, 1989), let us suppose that we have a generic theory $T$, and a set of $n$ ideal-condition claims: $I=\left\{I_{i} \mid i=1, \ldots, n\right\}$, such that $I_{j}$ is less idealized (or more realistic) than $I_{i}$, if $j>i$. For all $I_{i}$ 's, we have $I_{i}: T \Rightarrow T_{i}$, and $T_{i} \mid=P_{i}$, where $P=\left\{P_{i} \mid i=1, \ldots, n\right\}$ is a set of predictions. Suppose again that the non-idealized theory, $T^{U}$, predicts the true $P^{U}$. And let $\Delta^{-} I=\left|I_{j}-I_{i}\right|$ and $\Delta^{+} P=\left|P_{j}-P_{i}\right|$ (where $j>i$ ) denote the factual distance between two idealized conditions and two predicted states of affairs, we then have,

[A] $\mathrm{T}$ is a good idealized theory if it is always true that $T: \Delta^{-} I \Rightarrow \Delta^{+} P$; otherwise, it is not a good idealized theory.

In other words, when $I \rightarrow 0$, we have $T \rightarrow T^{U}$ and $P \rightarrow P^{U}$, if $T$ is a good idealized theory. This is how the measure of idealization is given by approximation approbation. Here the notion of factual distance, such as $\Delta^{+} P=\left|P_{j}-P_{i}\right|$, is not something easily definable in general; but when 
dealing with quantitative statements about singular states of affairs, the distance is supposed to be (Niniiluoto 1984) the distance of an Euclidean metric.

The theory and its variations are not so much wrong as are too simplistic in their depiction of idealization. For some limited domain of applications, Laymon's theory is just right, but if one looks at the whole range of things for which idealizations are needed -- a major portion of which are for discovering scientific laws via the construction of models -- one should realize that approximation approbation does not even come close in covering them. To show this I here give one example (see Liu 1999 for more).

Suppose that we are in Newtonian regime so that Newton's law of gravity is the law, i.e. $F=G(m M) / r^{2}$, for two objects with masses, $m$ and $M$, and separated by a distance of $r$. Let us see which of the following is more approximate.

[B] It is a law that given I, $F=G(m M) / r^{2}+a$;

and

[C] It is a law that given I', $F^{\prime}=G(m M) / r^{2}+b \sin (\omega r)$, where $|b|<|a|$ and $\omega$ is a frequency term.

If the factual closeness -- approximation approbation -- is the measure, $[\mathrm{C}]$ is a better fit than $[\mathrm{B}]$, especially if the frequency term, $\omega$, is large. While [B] consistently misses the law by $|a|$, [C] not only misses less -- $|b|<|a|$-- but also has frequent 'hits' on the correct values because of $\sin (\omega r)$. Hence, $\mathrm{F}^{\prime}$ given I' is a better idealized theory than F given I. But we all know that $[\mathrm{B}]$ is a better 'approximation' qua law of Newton's law even though it is not so qua facts. This and many other examples show that the notion of a lawlike statement being close to but not the true law statement has not one but two dimensions, namely, the closeness to the form of the law in question and the closeness to the singular facts which follow from the law. ${ }^{1}$ They obviously do not always go together, and the evaluation of whether a lawlike statement is approximate may involve checking out the balance between the two dimensions. However, it is not clear at all how or whether a measure of such a balance can be defined in general for all approximate lawlike hypotheses. 
3. An analysis of idealized laws. Idealization, as mentioned earlier, is too complex a notion of theory construction to be captured in a single logical formulation -- as in Nowak's (1972) -- or by a simple connection to approximation production -- as in Laymon's. To see a few of its diverse applications we note, first, that many acts of idealization are indeed for neglecting the negligibles; for instance, making $\sin \theta=\theta$, when the swing angle $\theta$ of a pendulum is very small, or taking some edge of a solid to be a continuous line. ${ }^{2}$ Second, idealizations play a big role in experimental data collection and analysis (cf. Kyburg 1990, ch. 10). Here, the idealized curves drawn among the data points, for instance, are the result of combined considerations of approximation and optimal statistical regularities. The third kind is one of the most important and richest category of idealizations whose sole purpose is, to use a catchy phrase, to carve nature at its joints. What scientists would like to do with nature in their theories is what that fabled butcher in Chuang Tzu can do with an ox: he can cut up (all the joints of), and so kill, an ox without anybody around him realizing it; not even the ox itself! (If someone but touches the ox, it will then collapse to the ground with all the parts in perfectly clean separation.) There is a fourth kind -- the class of limiting-case idealizations, which I will discuss later (cf. Liu 2001)

Let me leave the metaphor and begin to sketch a theory for idealization. But first, it seems better to call those abstractions which do nothing but to neglect the negligibles acts of approximation rather than of idealization. They are in fact so called by scientists. Now, our conception of idealizations as acts of coming up with idealized models in which idealized laws can be discovered and/or applied entails that we analyze first the notion of idealized laws and their relationship to the models. There are many competing theories of laws of nature, whose details and many controversies are beyond this paper. I shall take laws to be relations of natural necessity $^{3}$ among properties and are of the following two general forms (Armstrong 1983): It is a law that all $F \mathrm{~s}$ are $G \mathrm{~s}$, or $N(F, G)$,

and It is a law that for all the values in $P$ 's domain and all the values in $Q$ 's range, all $P$ s are $Q$ s where $Q=f(P)$, or $N[P, Q \mid Q=f(P)]$ 
(3) is what Armstrong calls the form of functional laws, which is the form the majority of lawlike statements in exact sciences take.

First, we need to know what kind of properties are admissible to, or relatable by, laws of nature. This, as we will see later, is crucial for the proper understanding of idealized laws. It seems quite safe to accept that which property is admissible to laws is ultimately for science to determine -- which however does not mean that it is only a matter of empirical findings. But some general questions may be settled independently of particular scientific inquiries (cf. Armstrong 1983; Lewis 1983). Some (e.g. Armstrong and Lewis) want to rule out dispositions and only allow categorical properties for laws. The reason for this exclusion of dispositional properties (including propensities) is multi-faceted. There is the Humean aversion to power, which -understood in a broad sense -- is what all the dispositional properties have in common; the idea that laws are first and foremost true regularities, which implies that the admissible properties ought to be occurrent to the systems which possess them; and the idea that dispositions are dispensable because they supervene on categorical properties, for example, the fragility of a vase is completely determined when all of its structural properties are; and the latter are categorical.

However, I find this restriction difficult to defend. I have mainly two arguments for the inclusion of dispositional properties (including the probabilistic ones). First, the categorists seem to take the fundamental magnitudes -- positions and momenta -- to be categorical, which uniquely determine the states of a system of particles in classical mechanics. But are they? Could the instantaneous velocity of a non-uniformly moving particle be considered a categorical property? I do not think it can. What the particle has at any instant is precisely the tendency of moving with the velocity it possesses at that moment but not actually moving with that velocity. The latter is a categorical property which it does not have, and the former -- which it does -- is a bona fide disposition. Here, the categorists seem to have been saddled in a dilemma: either they admit that momenta are admissible properties for laws of physics, and hence unwittingly admit that categorism is false; or they regard momenta as either illegitimate for physics or dispensable because they supervene on positions. They cannot really grab the first horn, but they cannot grab the 
second either; for either to deny the fundamental role of momentum in the description of physical states or to think that momentum supervenes on position is to reject physics, at least its classical portion, altogether. One of the most basic tenets of physics is that a system's degrees of freedom are determined by the independent components of the positions and momenta of its constituents -e.g. the $6 \mathrm{~N}$ phase space for a system of $\mathrm{N}$ particles. If momenta supervene on positions, the number of degrees should be cut in half. Theories, such as classical mechanics, may not be true, but they cannot be regarded as false in such an elementary way.

Second, there is a large class of lawlike hypotheses in exact sciences which relate nothing but dispositional predicates. That suggests that they are statements for dispositional laws -- laws relating dispositional properties. For instance, all laws expressed in the form of differential equations are laws of this kind. One may argue that the law that a differential equation represents is not the relation among the differentials (or derivatives), but rather that which is represented by the solution -- if the equation is solvable -- under certain initial and/or boundary conditions, which is in most cases a curve or trajectory of some sort. And the solution is indeed an equation relating categorical predicates (at least those for which the differential equation is solved). But it is a mistake to take in general the solutions rather than the differential equation as representing the law. The law of motion in e.g. Newtonian mechanics is represented by the differential equation relating the second-order time-derivative of a position function and the first-order (partial) space-derivative of a potential, not the infinite number and varieties of trajectories as the solutions of the equation under all the possible initial/boundary conditions. The categorists cannot even claim in this case that the law of motion comprise the set of all possible solutions of the equation of motion, because then the law is neither definable nor knowable. Therefore, I shall admit dispositions (and propensities) in my ontology, which we shall see is important in helping us to resolve a difficulty in understanding the nature of some idealized laws. ${ }^{4}$

We now turn to the discussion of idealized laws. By the end of this analysis we shall see that the admission of dispositions and a clarification of how certain type of laws are discovered or applied in idealized models help us to resolve the difficulty of how idealized laws may be true not 
only in their models but true simpliciter. I take an idealized law to be a law that obtains under certain stipulated conditions, which may be expressed by a hypothetical claim, such as 'if $I$, then (2)', where $I$ refers to the ideal conditions and see above for (2). First, one should note that I take this to be a 'hypothetical', rather than a mere material conditional or a counterfactual. It differs from a material conditional in that it is not automatically true whenever $I$ is false, for $I$ is false in most cases -- that's why it is a set of ideal conditions, but it is not a simple counterfactual, namely, if $I$ were the case, then it would be a law that all $F$ s are $G$ s. One may find this last claim puzzling, for is it not by definition that ideal conditions are those which do not obtain in the actual world? And if so, the counterfactual should be the right expression. This is, as mentioned earlier, how the traditional theories view ideal conditions. But I disagree; and I shall argue in detail in the next section that since idealization is a means for model-building in science, it is better to understand $I$ as hypothetical rather than simply false.

Second, one should also note that idealized laws are not the same as, or even a subset of, ceteris paribus laws (cf. Earman \& Roberts 1999). Many (Cartwright 1989; Hausman 1992; Silverberg 1996) have included idealized laws in the category of ceteris paribus laws, thinking that the ideal conditions are the ceteris that must be paribus in order for the corresponding law to hold. Earman and Roberts want to exclude idealized laws mainly for two reasons: (i) while the ceteris paribus conditions in a hedged law must actually obtain sometimes, the ideal conditions are by conception never actually obtain; and (ii) while the ceteris paribus conditions are usually not precisely and completely statable, the ideal conditions usually are. Because of what I have just said -- treating ideal conditions as hypothetical rather than non-actual better agrees with what scientists regard such conditions when they use them to build idealized models -- I cannot accept (i). I however think that (ii) is correct, because holding ceteris paribus law, such as those in social sciences, is precisely holding a law without an explicitly specified model, which is contrary to what happens with holding an idealized law. Some ceteris paribus laws, if there are any such laws, may involve idealizations; for instance, the ideal condition of having perfectly rational agents is perhaps assumed by many such laws in social sciences. But the ceteris paribus conditions are 
usually not such conditions. In other words, a law may at the same time be idealized and a ceteris paribus law, but its ceteris paribus conditions cannot be regarded as its ideal conditions.

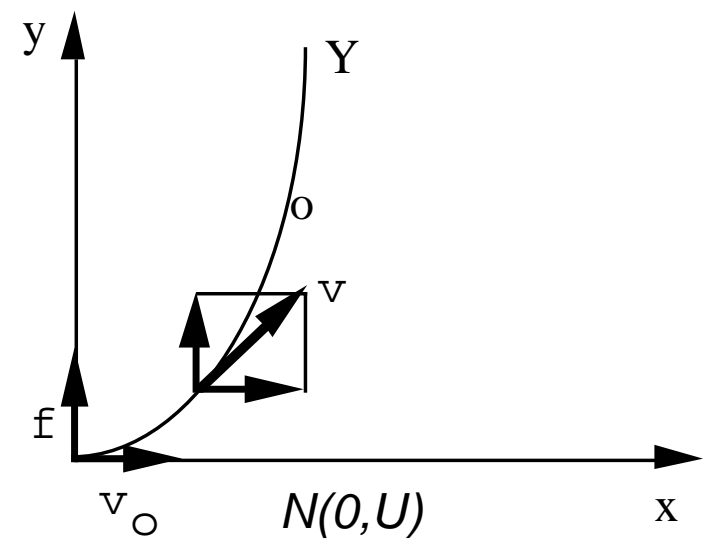

(a): $O=$ being an object with an initial velocity, $\mathbf{v}_{0}$, at $t=0 ; U=$ moving with that velocty along the $\mathrm{x}$-axis.

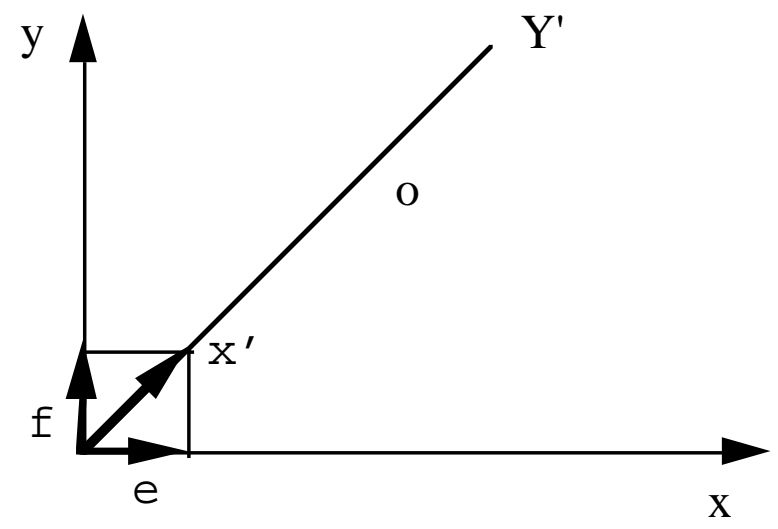

(b)

Figure 1: (a): a situation for (4), (b): a situation for (5). 


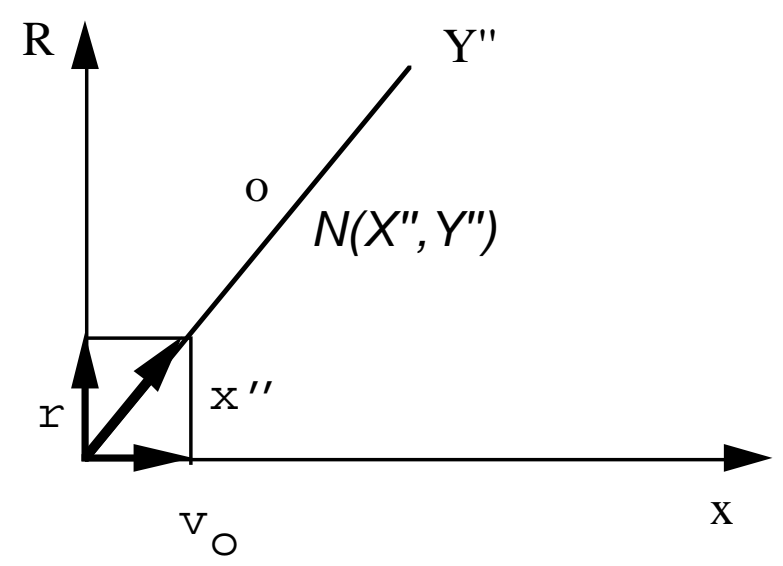

Figure 2: A hot body moving and radiating energy at the same time. $X^{\prime \prime}=$ being moving with $\mathbf{v}_{0}$, at $t=0$ and hot with $\mathbf{r} ; Y^{\prime \prime}=$ having moved a distance of $\mathbf{v}_{o} t$ and having radiated an amount of energy $R=\mathbf{r} t$, for some $t>0$.

Let us now see what idealized law hypotheses express. Without $I$, we have lawlike statements in the form of (2) which tells us that in every naturally possible world all $F$ s are $G$ s. There are plenty of such statements which if true are laws. 'All human are mortal', 'all material objects have mass', and 'whenever an object is affected by a force with a nonzero net, its behavior is altered in some way', and ...; all these if true are said to express exceptionless or covering laws. ${ }^{5}$ What then is the case for the following example:

(4) It is a law that all objects move uniformly on rectilinear trajectories?

The conventional wisdom says that it is not true in the actual world and therefore is not a law unless it is fitted with a clause which says 'given they are not affected by any non-zero external force'. Then it expresses an idealized law, a law that is only true with the provided condition (cf. Cartwright 1983, 1989; also Kyburg 1990, ch. 10)). Figure 1(a) gives a possible situation in which (4) is not true. ${ }^{6}$ There, the inertial motion of an object, $o$, with initial velocity $\mathbf{v}_{0}$, is affected by a force, $\mathbf{f}$, so that it ends up having Y (= a curved trajectory of such-and-such a shape). The following seems another example of the same kind: 
(5) It is a law that whenever an object of a mass, $m$, is acted upon by a force, $\mathbf{f}=m \mathbf{g}$, it moves with an acceleration, $\mathbf{g}$.

Similarly, this is said to be true only to an object under the condition that no other forces affect it. Figure 1(b) gives a possible situation in which (5) is not true. When another force, $\mathbf{e}=\frac{m}{e} \mathbf{a}$, is also acting on o, it fails to move in the direction of $\mathbf{g}$. Instead, it moves along the direction of $\mathbf{x}$ (or ends up having $\mathrm{Y}^{\prime}$ ).

Some, such as Cartwright $(1983)^{7}$, have argued that these, as idealized lawlike statements, cannot be true even though they may have enormous explanatory power, while many true law statements -- such as the covering laws given above -- do not explain at all. And yet one cannot deny that our intuition strongly suggest that (5) can be true without the idealization while (4) perhaps cannot. Is such an intuition simply false? Now, why is (4) apparently false without the ideal condition? Because if so, an instance of (4) as given in Figure 1(a), i.e. $N(O, U)$, would be true, where $O$ expresses the property of being an object with an initial velocity, $\mathbf{v}_{0}$, at $t=0$, and $U$ the property of moving with that velocity along the x-axis. Obviously, $N(O, U)$ does not appear to be true since any object possessing $O$ in this case ends up having $Y$, namely, tracing out a curved trajectory as shown. One may say that for essentially the same reason (5) is also false in the situation of Figure 1(b). This is how I understand Cartwright's view on such idealized laws. However, there is a difference between (6) the property of having described a trajectory of a certain shape,

and

(7) the property of tending to move at $t$ with a $\mathbf{v}$ and/or a towards a certain direction. (6) and its likes are full-bodied categorical properties while (7) and its likes can be seen as dispositions. If (5) is understood as a law which relates the actions of forces to dispositions of motion, it is possible to regard (5) as true in Figure 1(b): at every moment of its motion, the object, being acted upon by $\mathbf{f}$ (and also by $\mathbf{e}$ ), has the disposition of moving with $\mathbf{g}$. It is only when one demands that the admissible properties, at least in such cases as regarding an object's motion, be categorical do we run into the problem, and such laws as (5) become false without the ideal 
conditions. Hence, whether one should agree with Cartwright on the truth of such laws depends on whether one admits dispositions as possible relata of laws.

To further clarify the situation, let us see another case of co-instantiated properties where it is clear that no relevant idealization clauses are needed. In Figure 2, we have a hot body moving inertially along the $\mathrm{x}$-axis and radiating heat energy at the rate of $\mathbf{r}$. Suppose that two laws govern the behavior of such objects: one is (4), and the other a law: $R=\mathbf{r} t$. Supposing again that uniform motion and heat radiation do not affect each other, we should have no qualm in asserting that the two laws are true in this situation and true with no exception. By the end of a time interval, the object describes a certain distance along $\mathrm{x}$-axis and radiates a certain amount, $\mathrm{R}$, of heat energy. If we compare this case with the case of (5) in Figure 1(b), we could not help making the following observation. If there is a law in Figure 1(b) which is obviously true without exception among the relevant factors, it should be $N\left(X^{\prime}, Y^{\prime}\right)$ where $X^{\prime}=$ being acted upon be a force, $\mathbf{X}^{\prime}$. For this law, whether one use $Y^{\prime}$-- which is categorical -- or an dispositional equivalent, the law is true without exceptions. However, it seems odd to think that there also is a non-idealized law, $N\left(X^{\prime \prime}, Y^{\prime \prime}\right)$, in the case of Figure 2, where $X^{\prime \prime}=$ being moving with $\mathbf{v}_{0}$ and hot with $\mathbf{r}$ at $t=0$, and $Y^{\prime \prime}=$ having moved a distance of $\mathbf{v}_{o} t$ and radiated an amount of energy $R=\mathbf{r} t$, for some $t>0$. One must realize that since $\mathbf{v}_{\mathbf{0}} t+\mathbf{r} t=\left(\mathbf{v}_{\mathbf{0}}+\mathbf{r}\right) t=\mathbf{X}^{\prime \prime} t, N\left(X^{\prime \prime}, Y^{\prime \prime}\right)$ is a true generalization if each of the two statements of inertia and of radiation is separately. What worries us is the consequence of an unnecessary proliferation of laws: presumably any conjunctions of any number of co-instantiated (or co-instantiable) causal laws will give rise to new and distinct laws.

Let us now further analyze the three examples in terms of how different types of properties are co-instantiated and what results such co-instantiations produce. ${ }^{8}$ In Figure 2, every moment since $t=0$ sees the instantiation in the object, $o$, of the properties of being an object and of moving in its initial velocity and of being hot and of radiating energy. And any complex properties consisting some or all of these four properties are also instantiated in $o$ at that moment. The two laws, one relating being an object and moving in its initial velocity and the other relating being hot and radiating heat energy, hold separately on $o$, because the co-instantiations of the antecedent 
properties -- being an object and being hot -- on $o$ causes the co-instantiation of the consequent properties -- moving at some constant velocity and radiating heat energy. We can generalize this result to all laws that relate compatible categorical properties and to any number of their coinstantiations; the laws, if they hold, hold without exception in all such circumstances. And for the discovery and/or application of such laws, idealization is rarely needed, even if approximation is unavoidable. Such laws include causal and non-causal laws; for the latter we have those synchronic laws that determine the states of a system.

In Figure 1(b), every moment since $t=0$ sees the instantiation in $o$ of the properties of being affected by a force $\mathbf{X}^{\prime}$ and of moving with an acceleration, $\mathbf{c}=\mathbf{g}+\frac{e}{m} \mathbf{e}$. Since these two properties may be regarded as complex, the two pairs of properties -- being acted on by an $\mathbf{f}$ and by an $\mathbf{e}$, and moving with an acceleration of $\mathbf{g}$ and of $\frac{e}{m} \mathbf{e}--$ are also instantiated. Even though one cannot say that after time $t>0 o$ has in fact moved a distance of $\frac{1}{2} \mathbf{g} t^{2}$ and a distance of $\frac{e}{2 m} \mathbf{e} t^{2}$, one can say that at each moment it moves with $\mathbf{g}$ and it moves with $\frac{e}{m} \mathbf{e}$, because it is indeed true that at that moment it has the disposition of moving with $\mathbf{g}$ (or with $\frac{e}{m} \mathbf{e}$ ) regardless of where and how the other force is directing it to move. Although the final 'categorical' results over time cannot be a combined display that is decomposible into individual displays, the dispositions are uniquely and exactly decomposible at each moment. Therefore, if the two force laws are taken as laws relating dispositional properties, they are equally invariant laws, just as the ones in Figure 2 , and are both true in the circumstance.

Here the relationship between accelerations (dispositional) and trajectories (categorical) is the typical relationship between a disposition and its categorical display. For instance, a fragile body -- one that possesses the disposition of fragility -- displays its fragility when it is shattered -possessing a set of categorical properties -- after being subjected to a set of prescribed conditions. Similarly, an accelerated body displays its acceleration by describing over time a certain trajectory after being subjected to a set of initial and/or boundary conditions. In many cases, a coinstantiation of dispositional properties produces a 'combined' display that is not the simple sum 
of, or not decomposible into, the separate displays, each of which belongs to one of the coinstantiated disposition, such as the case in Figure 1(b). However, the combined display are predictable, and even derivable, when rules or schemes are provided by the relevant theories. In mechanics, the combined effect which displays the co-action of forces is precisely derivable via the mathematical techniques of linear algebra.

This result can be generalized to all laws that causally relate dispositions, at least when the causes and their joint effects are unambiguously decomposible. In this respect, there is no good reason to believe that such laws are fundamentally different from the ones we discussed above. And now, dealization is essential in the discovery of such laws through decomposition. It is only when other laws and the properties they relate are 'idealized away' can we find out the desired law in its full display. More on this later.

In Figure 1(a), every moment since $t=0$ sees the instantiation on the object the properties of being a object that moves initially at $\mathbf{v}_{0}$ and of being acted upon by a force $\mathbf{f}$ and of moving in a velocity $\mathbf{v}$. After some time $t>0$ it also has the property of having moved a distance of $Y$. The motion of the object at each moment is completely determined in this case by two dispositions: the inertial tendency to moving with $\mathbf{v}$ and the tendency to accelerate with $\mathbf{g}$ (which changes $\mathbf{v}$ through time). With them are instantiated two laws: the law of inertial -- as in $(4)^{9}--$ and the law of gravity -- as in (5), and each is true regardless of the other: if $\mathbf{f}$ is removed at some moment, the object will move with $\mathbf{v}$ from that moment on in accordance with (4), and the same is true if $\mathbf{v}_{0}=0$, mutatis mutandis. This is an example of a kind in which co-instantiated universals change each other over time, so that even though there are unique decompositions into sets of the same kind of properties at each moment in the process, the properties change -- i.e. having different properties at different times -- over time.

Here we have the co-instantiation of two laws in which the co-instantiations of the respective dispositions produces not only a non-decomposible effect but also a change in some of the dispositions over time. However, the combined displays may still be predictable or derivable, even though the calculation may be more complex, such as the case of Figure 1(a). 
4. The role of idealized models. With the above analysis of idealized laws -- or, better, laws under idealizations -- we can now return to discuss the notion that idealization is to carve nature at its joints. At this point, one may have the impression that the laws I discussed in section $\mathbf{3}$ and their likes exhaust the category of idealized laws. Given what I have said at the beginning of that section, this is far from the case. Moreover, there appear to be two other important classes of idealized laws that do not fit into what I have discussed so far.

There are those laws which are the results of idealizations on the structures of the systems in which they operate. Examples include Boyle's law of ideal gas, mechanical laws of rigid bodies, hydrodynamic laws of continuous fluids, etc. (cf. Kyburg 1990, ch. 10) And there are those laws which are the results of idealizations on the domains (or regimes) of their application. Examples include all the non-quantum and/or non-relativistic laws which can be seen as the limiting cases of the quantum and/or relativistic laws; for instance, laws in Newtonian mechanics can be seen as idealized laws in the regime of $v / c \approx 0$ and $\mathrm{h} / \varepsilon \approx 0$, where $v$ and $\varepsilon$ are, respectively, the speed and energy of the system in question.

Despite their apparent differences and the fact that scientists regard them as of different classes, I believe they really belong to the same category. Is Boyle's law not the 'limiting case' of van der Waals's law, where the limiting case may be understood as $a \rightarrow 0$ and $b \rightarrow 0$ in the van der Waals equation: $\left(p+a / v^{2}\right)(v-b)=k T$, which results in the Boyle equation: $p v=k T ?^{10}$ In practice, when the gas is diluted and at a high temperature, Boyle's law -- just like Newton's law of motion in contrast to the law of motion in special relativity -- is a good approximation. In both cases, the van der Waals equation and the special relativistic law of motion are 'truer', rather than true, law statements. In general, it is plausible to understand the idealizations taken on the structures for such laws as taking 'limiting' values of the relevant (or salient) structural properties of the model systems in question. And so, we can also understand the idealized structures in a model as the 'limiting' regime in which a simplified but still approximate version of a true or truer law statement holds. 
In contrast to the kind of causal/dispositional laws we just discussed, these idealized laws and their models do not seem to be the results of separation or disentanglement of processes or systems. Hence, the image of carving nature at its joints does not seem to apply to them. Also the idealized laws in these two classes are false without their idealized models. Further, there are no rules or schemes via which we can predict any true effects of any co-instantiations of such laws; whatever we can predict or derive from them must be at most approximately true.

Therefore, we may conclude that there are mainly two types of idealized laws whose discovery and application requires serious acts of idealization. One comprise the causal/dispositional laws I examined in the previous section, and the other limiting-case laws. For the latter, the situation is relatively simple, because they must be the limiting cases of some known true or 'truer' laws. They approximate the latter both in terms of the forms of the laws in question and of the singular factual consequences that follow from the laws. Recalling our discussion at the end of section 2, we now see that here is a kind of false lawlike hypotheses whose degrees of approximation to the true law statements can be determined with a relatively precise measure. And the measures are not, nor should they be, determinable by an epistemological theory; instead, they are given by the theories or disciplines in which the laws appear and within which the approximation of the laws is to be evaluated. ${ }^{11}$

In the practice of model-building in science, the two types of idealization may be employed at the same time for the study of a phenomenon or in the discovery of a law. In order for Newton's law of gravity (not in the form it is used in Figure 1(b), which is an even more simplified version of Newton's) to be true and fully displayed, an isolation of gravity from the action of other forces is not sufficient; one must also assume that the gravitational force in question is sufficiently weak, so that we are in a regime of 'weak-field' limit. And this situation is in fact quite common in all branches of sciences, where most of the laws we now have are likely to be only approximately true. It should be quite clear that what are responsible for the falsity of such laws are not the idealizations that disentangle or detach but those that set up the limiting-case regimes. 
We now return to our discussion of the dispositional laws and their models. A large number of laws in science belong to the types of the two examples (i.e. (4) and (5)) discussed earlier. Unlike the idealized laws as limiting cases, these laws are 'idealized' not in the sense that they themselves are unreal or not true or only approximately true but in the sense that to display them in their pure form one usually needs to construct idealized models. These are certainly not ceteris paribus laws, if by such laws we mean laws that are not true except under the ideal conditions. In this respect, I disagree with Cartwright (1999, 25-26, 28-29) who consider such laws as typical ceteris paribus laws, namely, they are not true unless the ceteris are paribus. (In the next section, I shall explain where the disagreement really lies.) This is because, as argued above, the properties -- in most cases dispositional ones -- the laws relate are instantiated fully in those non-ideal circumstances and thus the laws obtain. What is not always true for such laws when they are co-instantiated with other such laws is that they can show themselves in their full categorical display. Therefore, idealized models (or ideal conditions) are necessary for the laws to show their full categorical glory. In a staged drama where imaginary situations 'occur', certain typical characters which daily drive all of us to commit certain kinds of acts show themselves in their 'true' -- which means fully displayed -- color. It is no coïncidence that such characters are also dispositional properties of humans.

Modeling in science, whose essential means is idealization, does a similar job. The need of idealization and its difficulties increase when we go from those type of cases as in Figure 2 to those as in Figure 1(a). Little idealization if any is needed to discover the kind of laws operating in cases such as in Figure 2 because the antecedent and consequent universals, especially the latter, are separately instantiated categorical -- and separately observable -- properties. In other words, what are joined are joined rather obviously and loosely. The situation is different in the case of Figure 1(b) and its likes. It would not be so absurd for someone to argue that the law operating in this situation is really not the one for $\mathbf{f}$ or for $\mathbf{e}$ but the one for $\mathbf{X}^{\prime}$; and one does not need any idealization -- given these are the only two relevant forces -- to discover this law. But one needs idealization to discover that in this case two laws are in operation and the result is a co-instantiation 
of the two (recall Galileo's effort in separating friction from natural motion). In other words, $N\left(X^{\prime}, Y^{\prime}\right)$ expresses a mere regularity between $\mathrm{X}^{\prime}$ and $\mathrm{Y}^{\prime}$ as a result of the combined operation of the two laws. One may be skeptical about this conclusion, but one can be so only at the risk of doubting what most scientists have been doing for centuries (at least since Galileo's time when he inaugurated the method of idealization (cf. McMullin 1985; Suárez 1999)). It is precisely this kind of 'disentanglement' of simple (or simpler) processes, or 'detachment' of simple (or simpler) parts, from actual phenomena that produces models in which invariant laws can be found, whose co-instantiations in different combinations produce the great variety of empirical or phenomenological regularities we see in the actual world. And if one wants to know whether certain law is indeed operating in a mixture, one imagines ideal conditions in which the law operates relatively alone -- not that nothing else co-instantiate but rather that the situation be like in Figure 2.

Models are imagined in this fashion and experiments are often constructed to approximate the modeled circumstances. Here is the real connection between idealization and approximation. One must realize that not all good idealizations in science are directly approximatable; many have to be reduced via theoretical reasoning to some less abstract or more phenomenological situations where approximation in experiments are possible. We all know that whether a play reveals to us the true color of some characters does not depend on the verisimilitude of the circumstances designed to show them in the play (realistic plays are not necessarily the best ones in that respect). The merit of an idealization is rather measured in how well it offers the circumstances in which properties admissible to laws are easily separated and identified.

In sum, idealizations are necessary for the discovery and/or application of both types of laws of nature: the causal/dispositional laws and the limiting-case laws. But for the former they are mainly for discovery and display, while for the latter they really provide the truth-conditions (or the truth-regimes) for the laws. The inference is unsound which goes from the observation that all such idealized laws need idealized models for their discovery and/or application to that the laws must be false except in their models. It might be possible to conceive both types of idealized 
models as results of carving nature's joints, although it may not be a good fit. For the former, the carving consists of locating the places in nature that different component processes or systems join and detaching such components into idealized models. As I explained earlier, some of such components may actually exist in isolation, but typically they do not, and hence the need for idealization. For the latter, the 'carving' might be understood as an act of isolating proper levels of stable phenomena. In thermodynamics and hydrodynamics, the stable macroscopic phenomena are shown to us by nature because of the rarity of large fluctuations. The idealization may be seen as an act that ignores the discrete nature of bulk matter in these sciences and therefore theoretically insulates us from the microscopic world. And so does the main idealization for the ideal gas model -- i.e. the sizeless and forceless molecules -- for the diluted gases at high temperature. However, the notion of joints is no longer appropriate here and, because of it, the notion of recombination of different levels or domains does not make any sense.

5. Of laws, capacities and dispositions. In this section, I examine my proposal for understanding models and laws under idealization in contrast to theories of laws and related notions by Armstrong and Lewis and by Cartwright. The fundamental, and perhaps the only relevant, difference between my conception of laws and that of Armstrong's and Lewis's is that I admit non-categorical properties whereas they do not. Although Armstrong and Lewis disagree on what laws of nature are -- Armstrong takes them to be relations among universals and Lewis takes them to be the axioms of a system of regularities which has the best balance of strength and simplicity -- they agree on the categoricity of admissible properties. Hence, I am not a Humean; but nor is Armstrong; however, he and I are non-Humean of different kinds.

Then, one may argue that I would be thus barred from using Armstrong's theory of laws since it seems that I have to give up one of the three basic assumptions of Armstrong's theory -i.e. actualism (Armstrong 1983, 7-9). For Armstrong's actualism 'debars us from postulating such properties as dispositions and powers where these are conceived of as properties over and above the categorical properties of objects (ibid. p. 9).' Two observations on this point are in 
order. First, dispositions (and propensities) -- at least those ones which find frequent uses in physical sciences -- can be just as actually possessed by an object as its categorical properties are. The fragility seems to be just as good an actual property for a vase to have (whenever it is indeed fragile) as the property of its actual shape or mass. One should not confuse the disposition itself with the categorical properties which constitute a display of it and which are connected to it by some laws under a set of circumstances. Such properties, which an object only has the tendency to possess whenever the right circumstances arise, are indeed not actually possessed by the object; but the disposition -- the tendency -- the object undoubtedly does possess. Even if dispositions supervene on categorical properties, which I have argued cannot be true in general, the point is no different.

Since what a disposition may supervene on must be a set of categorical properties that an object actually possess, the object actually possesses the disposition precisely because it actually possesses those categorical properties. Second, even if this is not acceptable, Armstrong has admitted that the actualism assumption is the weakest, and 'the most difficult and uncertain of [his] three assumptions (ibid. p. 9).' Therefore, I am not barred from Armstrong's theory of laws; and I can simply extend actualism to those dispositional properties that are admissible to laws.

There may indeed be a more dangerous challenge in this connection that I am probably not able to completely meet. If being sentimental is a disposition of someone, say, Bill, who has not displayed and will never display any emotions which show that he is such a man, then why could not being 'lawyerly' also a disposition of Bill, where Bill is not, has never been, and will never be, a lawyer; and yet he could have been one had the right set of circumstances obtained at some time of his life. In other words, being lawyerly is a modal property of Bill. The question is this: does it make sense to say that Bill, who is one of the most matter-of-fact man in the world and a banker all his life, actually possesses the disposition of being sentimental and lawyerly, just because (let us suppose) given the right conditions he could have become sentimental and a lawyer? This to me seems more of a question of whether or not all modal properties are admissible to laws of nature than a question of which, or which kind of, modal properties can be said to be actually possessed 
by an object. To the latter question, I do not even know where to begin to address it, while to the former question, I suspect that it is the same kind of question as which (categorical) properties are admissible to laws. Both Armstrong $(1978,1983)$ and Lewis (1983) have written extensively on this question, and it has now become quite obvious that not all properties are admissible relata of laws of nature. I suspect the same kind inquiry has to be carried out for modal properties so that a proper subset of them -- in which are included all the dispositional properties already in use in science -- may be set aside for laws.

Cartwright $(1983,1989,1999)$ also rejects the Humeanism mentioned above (i.e. there are laws but no dispositions) and argues that we should also, and more importantly, admit capacities. Capacities are an entirely different concept from dispositions (or propensities), according to Cartwright (1999, pp. 59 and 64), because while dispositions 'are tied one-to-one to law-like regularities (p. 59)' or are 'identified with [some] particular manifestations, (p. 64)', capacities are not. They are entirely general and 'can have endless manifestations of endless different varieties.(p. 64)' And most important of all, they are not bound by laws of nature, which do bind dispositions. This distinction is crucially to Cartwright's conception of laws. For some reasons, which is not clear to me, Cartwright chooses to remain a categorist (á la Lewis) when laws and their admissible properties are concerned (cf. Cartwright 1999, 49-50), for she maintains that laws are regularities among occurrent (i.e. categorical) properties. This explains why she believes that most laws of nature are ceteris paribus laws in the strong sense that they are false when the ceteris are not paribus. ${ }^{12}$ Moreover, when the ceteris are not paribus and the laws are thus inoperative, capacities take over, or fill out, what is previously thought the role for laws. So, what happens when a situation as in Figure 1(b) arises? The law of gravity is no longer true there because of the interference of the electric force, but gravity as a capacity is still in operation since the object is indeed affected by it, and the tendency to move along $\mathbf{g}$-- if it can be regarded as a capacity -- is also in operation since the object does tend to move that way at every instant when it is affected by the gravity. But all these operations no longer obey the law of gravity! 
What is it that connects the capacities so that they can be causally efficacious? Can such connections be anything but nomic (= lawlike) ones? They cannot be logical, nor can they be merely factual. In fact, I think Cartwright does consider them to be lawlike in some sense of the word, but not in the sense she is using: 'We may ask, though, "Are not the laws for describing the capacities themselves just further regularity statements?" But we know from chapters 3 and 4 that the answer to this is "no". (1999, p. 142)' This may be right, for it is perhaps not defensible to regard laws relating capacities and dispositions as regularities; but if what I have argued so far is correct, there is no reason to so narrowly construe laws as regularities among occurrent properties.

I do not pretend to have completely understood Cartwright's notion of capacity; but it seems obvious that they are dispositional rather than categorical properties. It also seems that capacities are essential to whatever possesses them -- i.e. they are the natures of things -- and are highly abstract or general. But if they are, as Cartwright demanded, not to be related by laws of nature, they are not the type of dispositional properties that are admissible to my ontology. But since Cartwright's notion of laws, as mentioned above, is such a restricted one, not relatable by laws in Cartwright's terms does not mean the same as not ralatable by laws in my terms. So, when I open the door for dispositions and propensities for laws, there is no sound general philosophical reasons for barring laws among capacities. There may or may not be good empirical reasons -- in terms of scientific practice -- to include laws of capacities, but I do not see how one could reject them on purely metaphysical grounds while accepting dispositional laws.

Therefore, I disagree with Cartwright's theory of idealized laws -- to which I obviously owes a great deal -- on two major points: a whole class of idealized laws are true, not true ceteris paribus, and laws connect (admissible) categorical as well as dispositional properties, perhaps even capacities which I see as a special type of dispositional properties.

6. Conclusion. Let me now give a summary of the main features of this theory of idealization which articulates a relationship between laws and the idealized models in which they are true and/or displayed. There are two main types of idealized models: one consists of models which insulate 
levels or specify regimes in which simplified laws (or theories) are true as limiting cases of the true or truer laws (or theories), and the other consists of models which disentangle or detach component processes or systems in which (true) laws -- mostly dispositional ones -- operate in their full display. A law statement may be the result of a combined use of these two types of models, and if it is false (but approximately true) outside the models, it is because of the use of a model of the former type, not one of the latter.

Why do we need idealization in the sense of carving nature at its joints? Few, if any, genuine properties are instantiated (or exhibited) alone or in separation in our complex phenomenal world -- where the meaning of 'instantiation in separation' is exemplified by the case in Figure 2 -and some are never so instantiated. To discover them and the laws relating them for the construction of scientific theories, one has to separate them, which means that one has to stipulate conditions under which they are displayed purely and simply. This is the work of idealization. Moreover, nature's joints are not always sharp: some properties may overlap in the sense, for instance, the extensions of 'cat' and 'dog' may not be completely clear of each other. Therefore, 'carving', as one of the chief tasks of science, is to disentangle and set boundaries, which yields properties that are candidates for the relata of laws of nature.

Does not this talking of carving up nature (rather than finding out what nature itself is) commit me to the cause of social constructionism? No, not really. I do believe that there are constructive elements in our understanding of nature (i.e. in our true theories). The reason is simply this: idealization is indispensable in theoretical science and the result of idealization is the construction of models that may not obtain in nature. Theoretical arguments and experimental devices are often used to approximate the idealized models, but the models may never be literally realized. Hence, science as giving a description of what nature itself is, taken literally, is a false image. On the other hand, I am by no means forced by my own view on idealization to social constructionism. In fact, the very fact that I take approximation and idealization seriously excludes me from that club: the very idea of social constructionism, however one finesses it, excludes the notion of approximation and idealization. If it is fundamentally up to us to construct a coherent and 
practical system of theories which does not have to answer to any specific features of reality, why should, and how could, there be anything to approximate or to be idealized away?

\section{References}

Armstrong, D. M. (1978). Universals and Scientific Realism, vol. 2: A Theory of Universals. Cambridge: Cambridge University Press.

Armstrong, D. M. (1983). What Is a Law of Nature? Cambridge, Cambridge University Press.

Cartwright, N. (1983). How the Laws of Physics Lie. Oxford, Clarendon Press.

Cartwright, N. (1989). Nature's Capacities and Their Measurement. Oxford, Clarendon Press.

Cartwright, N. (1999). The Dappled World: A Study of the Boundaries of Science. Cambridge: Cambridge University Press.

Creary, L.G. (1981). "Causal Explanation and the Reality of Natural Component Forces." Pacific Philosophical Quarterly 62: 148-157.

Earman, J. and J. Roberts, (1999). "Ceteris Paribus, There is No Problem of Provisos." Synthese 118: $439-478$.

Hilpinen, R. (1976). Approximate Truth and Truthlikeness. Formal Methods in the Methodology of Empirical Sciences Eds. M. Przlecki, K. Szanianwski and R. Wojicki. Dordrecht, Reidel, 19-42.

Kline, D.A. and C.A. Matheson, (1986). "How the Laws of Physics Don't Even Fib. " PSA 1986 Eds. P. D. Asquith et al. East Lansing, Philosophy of Science Association. 1: 33-41.

Kyburg, H.E. Jr. (1990). Science and Reason. Oxford: Oxford University.

Laymon, R. (1980). Idealization, Explanation, and Confirmation. PSA 1980 Eds. P. D. Asquith and R. N. Giere. East Lansing, Philosophy of Science Association. 1: 336-350.

Laymon, R. (1985). Idealizations and the Testing of Theories by Experimentation. Observation. Experiment and Hypothesis in Modern Physical Science Eds. P. Achinstein and O. Hannaway. Cambridge, MA, MIT Press. 147-173.

Laymon, R. (1989). "Cartwright and the Lying Law of Physics." Journal of Philosophy 86: 353372.

Lewis, D. (1983). "New Work for a Theory of Universals." Australasian Journal of Philosophy 61: $343-377$.

Liu, C. (1999). "Approximation, Idealization, and Laws of Nature." Synthese 118: 229-256.

McMullin, E. (1985). "Galilean Idealization." Studies in the History and Philosophy of Science 16: $247-273$

Mellor, H. (1971). The Matter of Chance. Cambridge: Cambridge University Press. 
Mellor, H. (1974). “In Defense of Dispositions”, The Philosophical Review, 83, pp. 157-81.

Mellor, H. and A. Oliver (1997). Properties. (Oxford Readings in Philosophy) Oxford: Oxford University Press.

Mill, J.S. (1874). A System of Logic. New York: Harper \& Brothers.

Niiniluoto, I. (1984). Is Science Progressive. Dordrecht, Reidel.

Niiniluoto, I. (1998). "Verisimilitude: The Third Period." The British Journal for the Philosophy of Science. 49: 1-29.

Nowak, L. (1972). "Laws of Science, Theories, Measurement." Philosophy of Science 39: 533548.

Nowak, L. (1980). The Structure of Idealization: Towards a Systematic Interpretation of the Marxian Idea of Science. Dordrecht, Reidel.

Suárez, M. (1999). "The role of models in the application of scientific theories: epistemological implications.” Eds. Morgan, M.S. and Morrison, M. (1999). Models as Mediators: Perspectives on Natural and Social Science. Cambridge: Cambridge University Press, pp. 168-196.

\section{NOTES}

$\dagger$ I would like to thank Jossi Berkowitz, Carl Hoefer, Michael Redhead, Julian Reiss, Sang Wook Yi for questions and comments when I gave an earlier version of this paper for a Popper Seminar at the London School of Economics. I want to give special thanks to Carl Hoefer, Peter Lewis, and Michael Redhead for reading the penultimate draft and giving me detailed comments.

${ }^{1}$ In this paper, I shall sometimes use the term 'law' to refer to both the law and the statement that represents the law when there is no danger of confusing about which is meant in the context.

${ }^{2}$ Note that in the former we subtract something, i.e. $\delta=\sin \theta-\theta$, while in the latter we actually add something to the edge to make it continuous; but for convenience I shall call both types of idealization neglecting the negligibles since adding things to fill up the vacancies can be seen as acts of removing the vacancies.

${ }^{3}$ If one is a physicalist, then natural necessity is the same as physical necessity; but if one is not, the natural may be broader than the physical. Psychophysical laws, if there are any, are natural but not physical.

${ }^{4}$ See Mellor 1971 and 1974 for arguments on the legitimacy of dispositional properties in laws of nature. Mellor maintains that all properties are dispositional, a view I do not share. I cannot see what one can make sense of the display of a dispositional property if one does not also have categorical properties. But, as Mellow rightly pointed out, dispositions can be possessed actually and nomic connections among them are no different from ordinary nomic connections, and dispositions (and propensities) may change through time in accordance with the laws which bind them (without ever being displayed), just like categorical properties do. 
${ }^{5}$ Cartwright (1983) has argued for the scarcity of covering laws, which I think is not quite true. It is perhaps true that covering laws are usually too general, as our examples seem to show, to be useful in scientific explanation; but it certainly not true that they are scarce.

${ }^{6}$ Here, the law of inertial in (4) should be understood in its more precise formulation: it is a law that for any object, if it is initially in a state of motion with $\mathbf{v}_{0}$, it will remain in that state.

${ }^{7}$ For criticisms of this view of Cartwright's, see Creary 1981, Kline \& Matheson 1986, and also Laymon 1989; and for Cartwright's response, see Cartwright 1983, pp. 54-73.

${ }^{8}$ My view of idealized laws of the kind discussed so far is in many respects inspired by Mill's view of laws and causation (cf. Mill 1874, pp. 229ff, 237ff, 266ff, 317ff). A discussion of the relation between Mill's view and mine calls for another occasion. It suffices here to point out that my view is by no means the same as Mill's. As a starter, I do not subscribe to Mill's idea of what a law of nature is. There are serious problems with reconciling Mill's professed (sophisticated) regularitist conception of laws of nature and his use of dispositions and tendencies in his theory of causal laws. This and other complications in Mill's theory of laws will be examined in another place.

${ }^{9}$ I may be charged here with a gross equivocation between the law of inertial which governs objects that move under no influence and an intrinsic disposition -- inertia -- that all massed objects possess in resisting any changes of their states of motion. This is an important point, which is what makes me uncertain of how exactly to categorize laws like (4). On the one hand, it is a law concerning the universal intrinsic disposition of change resistance that all massed objects have; but on the other hand, the traditional formulation of the law is about objects' being moving uniformly and rectilinearly unless being prevented from doing so. The latter cannot be true without the 'unless...' clause, while the former is true at every moment of every massed object with no conditions required. Since there are not many lawlike statements of this kind in scientific theories, I shall leave the analysis of this case as is.

${ }^{10}$ Here, we have the following: $p$ is pressure, $v$ volume per molecule, $T$ temperature, $k$ the Boltzmann constant, and $a, b$ are the two correctional constants, whose values are determined by experiments.

${ }^{11}$ For a detailed study of this type of idealized laws, see Liu 2001.

${ }^{12}$ I have explained in section $\mathbf{3}$ my view on the difference between ceteris paribus laws and idealized laws. Here I follow Cartwright's use of the former as closely as possible. 